\title{
On asymmetries in cross-modal spatial attention orienting
}

\author{
LAWRENCE M. WARD, JOHN J. MCDONALD, and DANIEL LIN \\ University of British Columbia, Vancouver, British Columbia, Canada
}

\begin{abstract}
In a previous study, Ward (1994) reported that spatially uninformative visual cues orient auditory attention but that spatially uninformative auditory cues fail to orient visual attention. This cross-modal asymmetry is consistent with other intersensory perceptual phenomena that are dominated by the visual modality (e.g., ventriloquism). However, Spence and Driver (1997) found exactly the opposite asymmetry under different experimental conditions and with a different task. In spite of the several differences between the two studies, Spence and Driver (see also Driver \& Spence, 1998) argued that Ward's findings might have arisen from response-priming effects, and that the cross-modal asymmetry they themselves reported, in which auditory cues affect responses to visual targets but not vice versa, is in fact the correct result. The present study investigated cross-modal interactions in stimulus-driven spatial attention orienting under Ward's complex cue environment conditions using an experimental procedure that eliminates response-priming artifacts. The results demonstrate that the cross-modal asymmetry reported by Ward (1994) does occur when the cue environment is complex. We argue that strategic effects in cross-modal stimulus-driven orienting of attention are responsible for the opposite asymmetries found by Ward and by Spence and Driver (1997).
\end{abstract}

Several recent studies have investigated the extent to which there are cross-modal interactions in spatial attention (Buchtel \& Butter, 1988; Butter, Buchtel, \& Santucci, 1989; Farah, Wong, Monheit, \& Morrow, 1989; Mondor \& Amirault, 1998; Spence \& Driver, 1996, 1997; Spence, Nicholls, Gillespie, \& Driver, 1998; Ward, 1994; Ward, McDonald, \& Golestani, 1998). In one of these, Ward presented evidence that spatially uninformative visual cues orient auditory attention whereas spatially uninformative auditory cues fail to orient visual attention. On the basis of this evidence, Ward concluded that the neural mechanisms underlying stimulus-driven visual and auditory attention shifts are not completely modality specific, and speculated that stimulus-driven attention shifts are based on supramodal representations of space (see Farah et al., 1989). Importantly, however, the asymmetry in the cross-modal effects observed in Ward's experiment implies that such a supramodal representation is sometimes dominated by the visual modality and that conflicts in auditory and visual spatial information in these situations are resolved in favor of the visual input.

Ward (1994) interpreted his cross-modal attention results in the context of cross-modal asymmetries in spatial perception. Humans and other higher organisms pos-

Supported by a grant from the Natural Sciences and Engineering Research Council (NSERC) of Canada to L.M.W. and an NSERC Predoctoral Fellowship to J.J.M. Correspondence should be addressed to L. M. Ward, Department of Psychology, University of British Columbia, 2136 West Mall, Vancouver, BC, V6T 1Z4, Canada (e-mail: lward@cortex. psych.ubc.ca). sess specialized sensory systems that transduce different forms of energy into what seem to be, initially at least, independent perceptual experiences. However, the brain integrates signals from the various sensory systems to produce meaningful multisensory experiences and to facilitate the processing of multimodal objects in the natural environment. Such integration can affect stimulus detection and identification judgments, but the most well known behavioral consequences of cross-modal integration involve spatial localization judgments (for a review, see Welch \& Warren, 1986). For example, the appearance of a visual stimulus strongly influences the perceived location of a concurrent sound source (see, e.g., Bertelson \& Aschersleben, 1998), giving rise to ventriloquism and other intersensory phenomena. By comparison, the appearance of an auditory stimulus has little or no influence on the perceived location of a concurrent visual event, implying that the cross-modal interactions in spatial perception are dominated by the modality with the highest spatial resolution.

Although the cross-modal asymmetry in attention orienting reported by Ward is consistent with cross-modal asymmetries in spatial perception (e.g., ventriloquism), the exact opposite asymmetry of stimulus-driven attention orienting has been found by others (e.g., Klein, Brennan, \& Gilani, 1987) and was reported most recently by Spence and Driver (1997). In the latter paper, Spence and Driver suggested that Ward's results might have been caused by stimulus-response compatibility effects and thus might have no implications for the understanding of spatial attention orienting. Consequently, Spence and Driver have argued in favor of separate auditory and visual attention 
mechanisms that are "linked" in such a way that auditory events engage the visual attention mechanism, whereas visual events fail to engage the auditory attention mechanism (for a review, see Driver \& Spence, 1998). Since the conflicting asymmetries inform quite different theories of spatial attention, particularly in terms of its modality specificity, resolution of the empirical conflict is critically important.

Spence and Driver's (1997) major criticism of Ward's (1994) study was that his results might have reflected response priming by the cue. This explanation is possible because subjects in Ward's experiment pressed left- and right-hand buttons depending on the side of fixation on which they saw (visual task) or heard (auditory task) a target stimulus. Thus, the facilitatory cue effects that Ward observed at the 100-msec stimulus onset asynchronies (SOAs) might have arisen from a tendency of the subjects to prepare a response in the direction of the cue. When the target appeared on the validly cued side of fixation, the required response would have already been partially prepared in response to the cue, resulting in a faster response than when the target appeared on the invalidly cued side. Previous research has shown that irrelevant spatial information can affect responses to lateralized stimuli under a variety of conditions (for a recent review, see Lu \& Proctor, 1995). For example, responses to the monaurally presented auditory messages "left" and "right" are faster when the content of the message is consistent with the ear that is stimulated than when it is inconsistent (e.g., Simon, 1968). Similar effects, herein referred to as Simon effects, ${ }_{1}^{1}$ have been documented in vision (Craft \& Simon, 1970), even when the irrelevant spatial signal appears in a different modality (Simon \& Craft, 1970).

Despite the fact that irrelevant spatial information can influence choice response times in many situations, it seems unlikely that response priming can explain Ward's (1994) pattern of cue effects for two reasons. First, in most studies of the Simon effect, the irrelevant spatial signal is a feature of the imperative stimulus itself or of a stimulus appearing simultaneously with it. These findings imply that subjects in Ward's task would have had a strong tendency to respond in the direction of the target stimulus, as the tasks demanded. It is unclear whether any tendency to respond in the direction of the prior cue could have affected the tendency to respond in the direction of the target. Bearing on this issue, one study found that a lateralized warning tone failed to affect responses to a subsequent target tone when subjects responded by pressing ipsilateral response keys (Simon, Acosta, \& Mewaldt, 1975). This suggests that any tendencies to respond in the direction of the warning stimulus were not strong enough to affect the responses to the subsequent target (but see Proctor \& Pick, 1998). Second, the auditory cue did not affect response times to the visual target in Ward's experiment, although it did influence responses to auditory targets. If response priming were the only factor affecting response times in his experiment, then auditory cues should have affected response times to visual targets.
Given that this cue effect failed to occur, Ward concluded that the results of his experiment did not arise from response priming. Clearly, any response-priming explanation of Ward's results would have to account for this null cue effect as well as the positive cue effects observed in other conditions.

Spence and Driver (1997) did suggest a possible explanation for the lack of auditory cue effect on response times to visual targets in Ward's (1994) study. They suggested that the auditory cues and visual targets might have produced competing response tendencies because the auditory stimuli were presented at greater eccentricities than the visual stimuli (the auditory stimuli in Ward's experiment were presented from speakers mounted at the sides of the computer monitor). For example, an auditory cue appearing on the left side of fixation would activate a leftward response, whereas a subsequent visual target appearing on the left side of fixation would activate a rightward response because it appeared to the right of the cue. The response tendencies generated on these trials would be similar to those generated on invalid-cue trials, thereby failing to produce strong cuing effects in the auditory cue-visual target condition of Ward's experiment.

One implication of this explanation is that any effect of the cues on attention orienting should have emerged in the auditory cue-visual target condition once the response tendencies canceled out. However, a significant cue effect did not emerge, suggesting that Ward's (1994) conclusion about the lack of auditory cue effect on response times to visual targets was essentially correct. This null cue effect has been replicated in several recent experiments in which the possibility of response priming by the cue was reduced (Ward et al., 1998). However, it is still possible to argue that response priming might have influenced other effects in Ward's study, possibly adding to the attention orienting effects in some conditions and subtracting from them in others, including the crucial visual cue-auditory target condition, in which Ward found a large cuing effect.

The major aim of the present study was to test the correctness of the asymmetry found by Ward (1994) under his experimental conditions. In order to do this, we used a technique that is immune to response-priming effects and that has been shown in other experiments to be effective for producing auditory spatial cue effects (which are the most problematic for cross-modal studies, McDonald \& Ward, 1999). In this technique, subjects are instructed to respond whenever a target occurs in one of the two peripheral locations but to withhold their response if the target occurs in the central location. We call this the implicit spatial discrimination task. Since the response is always the same - a press of a single button-it cannot be differentially primed by cues to one side or the other. Nonetheless, in order to make responses only on the correct trials, subjects must localize the targets before responding (or not) to them. A replication of Ward's cross-modal asymmetry under these conditions would challenge recent proposals that either there are no cross-modal interactions at 
A

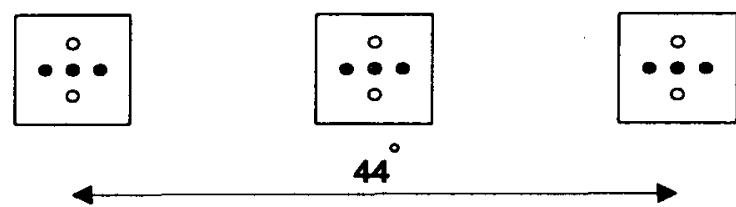

SPEAKER

○ TARGET LEDS

- FIXATION LED

- CUE LEDS
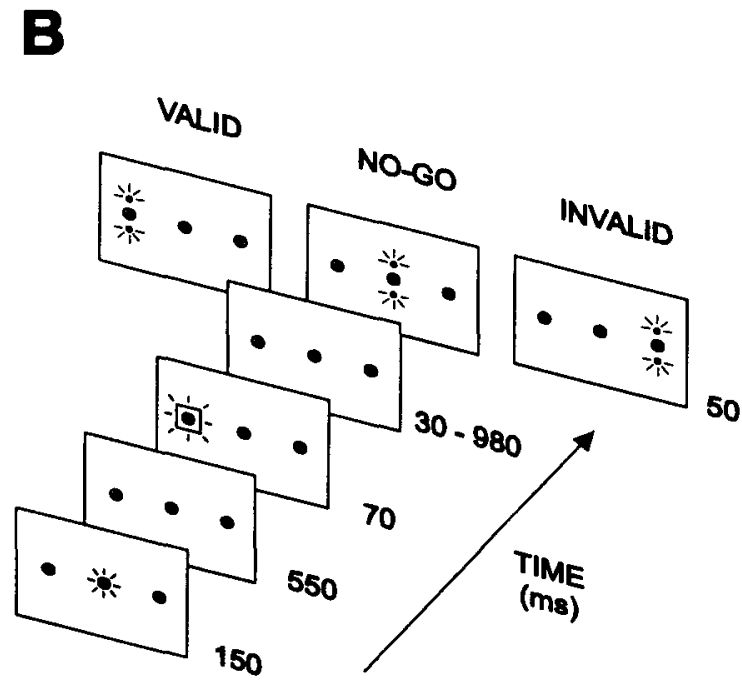

Figure 1. (A) Apparatus used to display auditory and visual stimuli in the present experiment. Note that visual and auditory cues and targets emanate from the same locations. (B) Sequence of events on a single trial in the experiment. An auditory cuevisual target trial is illustrated.

all in stimulus-driven spatial attention orienting (Mondor \& Amirault, 1998) or that such interactions are dominated by the auditory modality (Spence \& Driver, 1997).

\section{METHOD}

\section{Subjects}

Thirty-eight undergraduate students ( 15 male, 23 female) at the University of British Columbia, ages 18-23, were paid for their participation. Twenty subjects ran in the visual task and another 18 subjects in the auditory task. All subjects reported normal or correctedto-normal vision and hearing.

\section{Apparatus}

The experiment was conducted in a darkened, sound-attenuated chamber $(183 \times 193 \times 197 \mathrm{~cm})$ with a background noise level of $35 \mathrm{~dB}$. The chamber contained an adjustable headrest and a response box placed on a small table. Figure 1A shows the stimulus apparatus. Three horizontally aligned speakers were positioned so that the center speaker was $105 \mathrm{~cm}$ directly in front of the headrest.
The two peripheral speakers were placed at $22^{\circ}$ to the left and right of the central speaker. Five light-emitting diodes (LEDs) were mounted on each of the speakers. Green LEDs were placed at center (dim place holders that were always on) and to the left and right of center while red LEDs were placed above and below the center of each speaker. In both tasks visual cues consisted of a 70-msec flash of the (non-place-holder) pair of green LEDs on a particular speaker. Visual targets were 50-msec flashes of pairs of red LEDs on a particular speaker. Production of cue and target sounds was controlled by a custom sound generator. Auditory cues consisted of two successive $30-\mathrm{msec}$ broadband noise bursts $(2.5$-msec rise/fall time), separated by a $10-\mathrm{msec}$ silent interval and presented at $70 \mathrm{~dB}$. Auditory targets were $1000-\mathrm{Hz}$ pure tones presented at $75 \mathrm{~dB}$ for $50 \mathrm{msec}$. Low-frequency tones were presented whenever subjects responded erroneously and were easily distinguishable from both target and cue sounds and were of course presented immediately after a response. Subjects made their responses by pressing a single button that operated a microswitch. A custom input-output board with an interval timer chip was used to measure response latencies. Stimulus presentation, timing, and data acquisition were controlled by a microcomputer (IBM compatible) running custom software.

The horizontal electrooculogram (EOG) was used to monitor eye position throughout. Tin electrodes were placed $1 \mathrm{~cm}$ lateral to the left and right outer canthi. EOG activity was amplified with a bandpass of $0.1-30 \mathrm{~Hz}$ and continuously digitized at a rate of $128 \mathrm{~Hz}$. Electrode impedance was kept below $5 \mathrm{~kW}$. Trials on which eye movements occurred were discarded before analysis.

\section{Design and Procedure}

Figure IB summarizes the procedure. Subjects were instructed to keep their eyes fixated on the central LED of the central speaker at all times during a block of trials. All trials began with a brief flickering of the fixation LED followed by a 550-msec delay. A cueauditory, visual, both, or none - then appeared randomly from one of the three possible locations, giving rise to 16 different cuedefined conditions (as in Ward, 1994). Subjects were told that the cues were not predictive of target location. After a variable SOA $(100,200,550$, or $1,050 \mathrm{msec})$, a target was presented. Separate groups of subjects participated in the auditory and visual tasks, with either auditory or visual targets appearing on every trial, respectively. All subjects were instructed to respond as quickly and as accurately as possible when the target appeared on the left or right side of fixation (go trials) but to withhold their response and await the next trial when the target appeared at fixation (no-go trials). Subjects responded by pressing a single button with their dominant hand on all trials. To eliminate any possibility of response priming, subjects were not allowed to press the button with either hand. A 500 -msec error tone was presented if a response was made on a nogo trial or if no response was made on a go trial. The next trial began after a 1,500-msec intertrial interval.

Practice trials were performed until the subject was able to recognize cues and targets accurately. This was usually about 50 trials. Following the practice trials, subjects ran in three separate sessions. Each session consisted of four blocks of 128 correct-response go trials (plus about 26 no-go trials) for a total of about 1,850 trials (incorrect go trials were re-presented later in the block). Rest periods were given between blocks.

For both tasks, the conditions in which at least one cue appeared at a peripheral location were collapsed across left and right into six conditions for analysis as follows (Table 1): visual cue - visual cue from left or right, no auditory cue; auditory cue - auditory cue from left or right, no visual cue; combined cue - visual and auditory cues from same left or right location; opposite cues-visual cue from left and auditory cue from right or vice versa; visual/central-auditory cue - visual cue from left or right, auditory cue from center; auditory/ central-visual cue-auditory cue from left or right, visual cue from center. 
Table 1

Mean Valid (V) and Invalid (I) Response Times and Cue Effects (CE, Invalid RT - Valid RT) as a Function of Target Modality, Cue Condition, and Stimulus Onset Asynchrony

\begin{tabular}{|c|c|c|c|c|c|c|c|c|c|c|c|c|}
\hline \multirow[b]{3}{*}{ Cue } & \multicolumn{12}{|c|}{ Stimulus Onset Asynchrony } \\
\hline & \multicolumn{3}{|c|}{$100 \mathrm{msec}$} & \multicolumn{3}{|c|}{$200 \mathrm{msec}$} & \multicolumn{3}{|c|}{$550 \mathrm{msec}$} & \multicolumn{3}{|c|}{$1,050 \mathrm{msec}$} \\
\hline & $\mathrm{V}$ & I & $\mathbf{C E}$ & $\mathrm{V}$ & $\mathrm{I}$ & $\mathbf{C E}$ & $\mathrm{V}$ & $I$ & $\mathbf{C E}$ & $\mathrm{V}$ & I & $\mathbf{C E}$ \\
\hline \multicolumn{13}{|c|}{ Visual Target } \\
\hline Auditory & 393 & 404 & 11 & 365 & 374 & 9 & 376 & 381 & 4 & 387 & 382 & -5 \\
\hline Auditory/central-visual & 405 & 414 & 9 & 368 & 381 & $13^{*}$ & 359 & 370 & 11 & 356 & 359 & 3 \\
\hline Visual & 423 & 428 & 5 & 398 & 388 & -10 & 386 & 347 & $-39^{\star}$ & 396 & 356 & $-40^{*}$ \\
\hline Visual/central-auditory & 396 & 398 & 2 & 383 & 368 & $-15^{*}$ & 380 & 342 & $-\mathbf{3 8}^{\star}$ & 374 & 350 & $-24^{*}$ \\
\hline Combined & 393 & 397 & 4 & 378 & 367 & -11 & 380 & 335 & $-45^{\star}$ & 376 & 342 & $-36^{*}$ \\
\hline Opposite & 392 & 396 & -4 & 354 & 380 & $-26^{*}$ & 340 & 375 & $-\mathbf{3 5}^{\star}$ & 341 & 371 & $-30^{*}$ \\
\hline \multicolumn{13}{|c|}{ Auditory Target } \\
\hline Auditory & 455 & 493 & $39^{\star}$ & 419 & 447 & $28 *$ & 429 & 442 & 13 & 451 & 456 & 5 \\
\hline Auditory/central-visual & 491 & 543 & $52 *$ & 441 & 464 & $23^{\star}$ & 430 & 433 & 2 & 444 & 440 & -4 \\
\hline Visual & 486 & 516 & $30^{*}$ & 475 & 476 & 2 & 463 & 472 & 9 & 479 & 465 & -14 \\
\hline Visual/central-auditory & 496 & 504 & 8 & 441 & 450 & 9 & 430 & 434 & 5 & 435 & 439 & 4 \\
\hline Combined & 454 & 503 & $49^{\star}$ & 420 & 464 & $44^{*}$ & 422 & 428 & 6 & 443 & 432 & -11 \\
\hline Opposite & 471 & 471 & 0 & 425 & 432 & 7 & 420 & 426 & 6 & 439 & 438 & -1 \\
\hline
\end{tabular}

*Significant cue effect (cue effect in bold) at $\alpha=.025$ as measured by Bonferroni two-tailed $t$ test (see text for details).

\section{RESULTS}

Results from 3 subjects in the visual task and 1 subject in the auditory task were discarded without analysis because they made too many eye movements, leaving too few experimental trials for analysis after excluding the trials on which eye movements had been made. Approximately $7.7 \%$ of trials for the remaining 17 subjects in each task were discarded because of eye movements occurring within $1 \mathrm{sec}$ of the target onset. The average percentage of no-go errors was $7.6 \%$ in the visual task and $7.3 \%$ in the auditory task for these subjects.

Results of the "neutral" cue trials (both cues from center, only one cue from center [either auditory or visual], or no cues at all) were similar to those of Ward (1994) and are not discussed in detail here. Analysis was conducted on the subjects' median response times for the six conditions described in the Method section separately for each of the two experimental tasks. Table 1 displays the intersubject averages of these response times and the corresponding cue effects (invalid-cue response time minus valid-cue response time) for all SOAs. As in Ward's study, the average response times were longer in the auditory task ( $464 \mathrm{msec})$ than in the visual task $(386 \mathrm{msec})$. More significantly, the auditory cue had a minimal effect $(11 \mathrm{msec})$ on responses to the visual target at the 100 -msec SOA, whereas the visual cue had a large effect $(30 \mathrm{msec})$ on responses to the auditory target at the 100 msec SOA. This pattern of results essentially replicates the asymmetry reported by Ward ( $8 \mathrm{vs}$. $50 \mathrm{msec}$ for the auditory-on-visual and visual-on-auditory effects, respectively). The cue effects in the crucial cross-modal conditions are displayed in Figure 2.

A 6 (condition) $\times 4$ (SOA) $\times 2$ (validity) multivariate analysis of variance (MANOVA) (for repeated measures) was performed separately on the data from the two experimental tasks. All significance determinations were based on an alpha level of .05. All main effects and interactions were significant for both tasks; only the most directly relevant effects are reported in detail here. A main effect of SOA was found for both the visual and auditory tasks $[F(3,48)=15.12, p<.001 ; F(3,48)=38.15$, $p<.001$, respectively]. This result is usually interpreted as an alerting effect. As expected, there was an interaction between SOA and condition for both tasks [visual, $F(15,240)=7.62, p<.001$; auditory, $F(15,240)=4.74$, $p<.001]$, which we interpret as different alerting effects produced by the cues in different conditions. A significant interaction between SOA and validity was also found for both tasks [visual, $F(3,48)=17.02, p<.001$; auditory, $F(3,48)=14.74, p<.001]$, indicating that cue effects changed with SOA.

Bonferroni tests based on an experiment-wise error rate of .10 were used for planned comparisons (as in Ward, 1994) with the cue condition defining the experimental unit, so that four tests were done for each condition in each task (invalid-cue trials vs. valid-cue trials at each SOA; $p=.025$ for each). An asterisk in Table 1 denotes significant cue effects by this test. Again, the two critical cross-modal conditions are the auditory cue condition for the visual task and the visual cue condition for the auditory task because prior studies have found different patterns of results in those conditions. The relatively conservative Bonferroni analysis of the present results confirms Ward's conclusions: The present data indicate that under Ward's complex cue conditions, visual cues significantly affected responses to auditory targets, but auditory cues had only a small and insignificant effect on responses to visual targets. Since this analysis is somewhat conservative, and the $11-\mathrm{msec}$ effect only just missed being significant (the critical difference was $12 \mathrm{msec}$ ), we also performed a $4(\mathrm{SOA}) \times 2$ (validity) ANOVA on the auditory cue condition in the visual task only. This analysis revealed a significant main effect of SOA $[F(3,48)=$ 

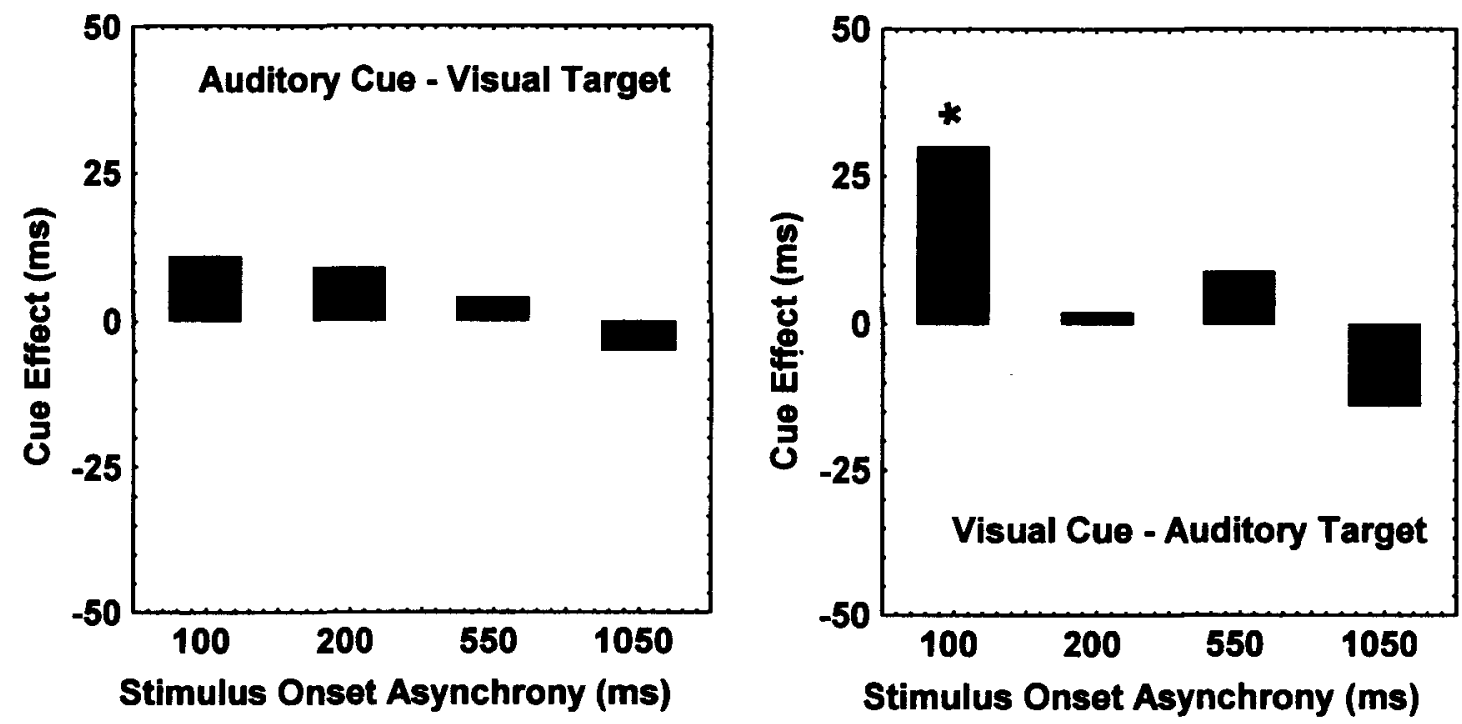

Figure 2. Cue effects for all SOAs for the crucial auditory cue-visual target and visual cue-auditory target conditions. An asterisk near a bar means that the cue effect is significant by Bonferroni $t$ test (described in text).

$9.21, p<.0001]$, but no significant main effect of validity $[F(1,16)=3.69, p=.07]$ and no interaction of the two $[F(3,48)=1.62, p=.20]$. Thus, if an effect of auditory cues on responses to visual targets was present under these conditions, it was not large enough to be reliably detected. Indeed, the size of the auditory cue on visual target effect is about the same in this experiment as in Ward's $(11 \mathrm{msec}$ here vs. $8 \mathrm{msec}$ in Ward), and only about one third the size of the highly significant effect of the visual cue on responses to auditory targets.

The cue effects reported in Table 1 also indicate some other interesting results of the present experiment, especially in relation to those of Ward (1994). First, the appearance of a visual cue did not facilitate responses to the visual target on valid-cue trials at the $100-\mathrm{msec}$ SOA in any condition involving a peripheral visual cue (visual cue, combined cue, opposite cue, and visual/centralauditory cue conditions). Instead, the appearance of a visual cue consistently inhibited responses to the visual target on valid-cue trials at longer SOAs in these conditions. These inhibitory effects, which are usually interpreted as inhibition of return (IOR), were substantially larger in the present experiment than those reported by Ward. Given the fact that significant IOR began as early as the 200-msec SOA in some conditions, either the time course of facilitation was too fast to be observed in this experiment, having already occurred and dissipated before the shortest SOA was over, or facilitation was overwhelmed by inhibition under these conditions. Tassinari, Aglioti, Chelazzi, Peru, and Berlucchi (1994) reported similar results in several experiments, with inhibitory effects of transient visual cues on responses to visual targets appearing at SOAs as short as $60 \mathrm{msec}$. In a review of these and other visual cuing studies, Tassinari and Berlucchi (1995) concluded that this is not an unusual finding when visual cue and target do not overlap in time. Such short-SOA inhibitory effects were attributed to sensory processes because they also occur with bilateral cues. In contrast, longer SOA inhibitory effects were attributed to aftereffects of covert orienting because they occur only with unilateral cues.

Second, and in contrast to the pattern of results obtained in the visual task, the appearance of a visual cue did facilitate responses to the auditory target on valid-cue trials at short SOAs but did not produce significant IOR at the longer SOAs. The facilitatory cue effects obtained in the auditory task were somewhat smaller than those reported by Ward (1994). However, cross-modal IOR failed to occur in the auditory task of both the present study and of Ward's study, with the exception of the combined cue condition of Ward (VAcon in Ward's terminology). The significant IOR observed in that condition was likely caused by the appearance of the auditory cue, which, unlike the visual cue, did produce significant IOR when presented alone. The lack of significant cross-modal IOR is consistent with recent results that indicate that cross-modal IOR is found only when the modality of the target stimulus is uncertain (McDonald \& Ward, 2000).

Third, the appearance of conflicting auditory and visual cues had very different effects on responses to auditory and visual targets in the present study. On the one hand, the appearance of a conflicting auditory cue had little or no influence on the IOR effects of the visual cue on responses to the visual target. These findings imply that the auditory cue did not influence the ability of the visual cue to generate visual IOR and are consistent with the proposal that the auditory cue did not orient visual attention or generate visual IOR. On the other hand, the appearance of a conflicting visual cue seems to have abolished the strong facilitatory effect the auditory cue had on responses to auditory targets when presented alone (auditory cue condition) or with a visual cue at the same location (com- 
bined cue condition). These findings imply that the visual cue did influence the ability of the auditory cue to orient auditory attention. We suggest that under these conditions both the visual and auditory cues were effective signals for orienting auditory attention, thereby facilitating processing of the auditory target when they occurred at the same location and inhibiting attention shifts when they occurred at different locations. The fact that there was no net cue effect on response times to the auditory target at the 100-msec SOA in the opposite cue condition indicates that the visual and auditory cues were roughly equally effective for orienting auditory attention (Table 1).

\section{DISCUSSION AND CONCLUSIONS}

The results of the present experiment replicate Ward's (1994) results in the crucial cross-modal conditions. This replication was accomplished using an experimental procedure that is immune to response-priming effects. We also monitored eye movements to ensure that they did not contaminate the results. ${ }^{2}$ Consequently, the asymmetry reported here cannot be explained in terms of response priming or eye movements. An explanation based on changes in the subject's criterion for responding also appears unlikely for several reasons. First, although the cues were the same in both experimental tasks, they had facilitatory effects only in the auditory task. There is no a priori reason why the visual cues should lower the criterion for responding to auditory targets appearing at their location but not for visual targets appearing at their location. A reviewer suggested that the response criterion might have been changed in both tasks, but the effect was countered by a masking effect of the cue in the visual task. However, visual cues and targets were selected so as to minimize such cue-target interactions (see Method section), which are anyway more likely to lead to short-SOA facilitation (Tassinari \& Berlucchi, 1995), so this does not seem plausible. Second, there were strong spatial cue effects on multiple-cue trials. Which cue would be the one responsible for lowering the criterion on such trials? Third, subjects couldn't use a simple decision rule like "be ready to respond to any target that occurs at a cued location," since the various cue conditions were randomly intermixed and significant cue effects were observed only for some of them. Thus, any changes in the criterion for responding would need to be made dynamically, after the appearance of the cue but before the appearance of the target. We doubt whether subjects could make such decisions when the interval between the cue and target was $100 \mathrm{msec}$.

It seems reasonable to conclude that, with regard to our spatial go/no-go task and under the conditions of a complex cue environment and certain target modality, visual cues strongly orient spatial attention so as to speed responses to auditory targets occurring in the same location relative to those occurring in other locations, but auditory cues fail to strongly orient spatial attention so as to influence responses to same-location visual targets. Although there are some differences between Ward's (1994) results and those of the present experiments, none of those differences affects this conclusion. In the face of the evidence for the opposite asymmetry provided by the data of Spence and Driver (1997) and others under different conditions, we are left with a need to understand what factors affect the ability of visual and auditory cues to orient spatial attention cross-modally.

One important factor appears to be the complexity of the stimulus conditions used in the experiment. In any spatial cuing experiment, the stimulus conditions can vary along several dimensions, giving rise to at least three separate categories of complexity. These dimensions include the cue modality (one or more), target modality (one or more), number of cues possible (one or more), and consistency of the cue locations (consistent, inconsistent, or mixed) to name a few. The least complex experiment would have the lowest value on each dimension (e.g., one cue modality, one target modality, one cue), whereas the most complex experiment would have higher values on each dimension (e.g., multiple cue modalities, multiple target modalities, multiple cues, possibly inconsistent cues). An experiment could also have lower values on some dimensions and higher values on others, creating numerous intermediate categories of complexity. The present experiment, like Ward's (1994) experiment, involved a complex cue environment and a simple target environment. In contrast, most of Spence and Driver's (1997) experiments involved a simple cue environment and a more complex target environment. Other recent experiments by McDonald and Ward (2000) used a simple cue environment and manipulated target environment complexity.

We believe that auditory cues fail to influence responses to visual targets when the cue environment is complex, as in Ward's (1994) and the present study, because subjects do not fully process the spatial location of the auditory cues under these conditions. When cues are uninformative and often conflicting and targets are all visual, it suffices to process the more directly encoded property of frequency content of the auditory cue in order to register it as a nontarget stimulus and use its appearance as an alerting stimulus to prepare for the target. The more laborious spatial location processing of the auditory cue is simply not done because it interferes with the subject's task. Thus spatial attention is not affected by the location of the auditory cue. Two sources of evidence support this conclusion. First, we have shown previously that spatially uninformative auditory cues influence responses to auditory targets only when the experimental task engages the proper spatial representations of the cue and target (McDonald \& Ward, 1999). Second, and more important, McDonald and Ward (2000) found that spatially uninformative auditory cues do orient both auditory and visual attention when both the cue and target environments are simple. Those experiments involved the same implicit spatial discrimination procedure as in the present study, differing only in the complexity of the cue environment. These findings imply that the present finding of a null effect of auditory cues on responses to visual tar- 
gets was caused by the complexity of the cue environment rather than by an unknown aspect of the implicit spatial discrimination procedure. Notice that this explanation predicts that a similar lack of effect of auditory cues in a complex cue environment would be found for responses to tactile targets, since the same reasoning would apply in that case. Such an experiment has not yet been done.

With respect to the asymmetry observed by Spence and Driver (1997), it is possible that the failure of visual cues to affect response times to auditory targets is specific to elevation discrimination tasks (Driver \& Spence, 1998; Ward et al., 1998). For example, when cue and target are relatively far apart on valid-cue trials, as they are in the elevation task, attentional effects can be expected to be weak. In particular, the spatial focusing of attention in response to visual cues might be too narrowly distributed at the cued location to affect the processing of distant auditory targets, producing little or no spatial cue effect. In comparison, the spatial focusing of attention in response to auditory cues is probably more broadly distributed because of the poorer spatial resolution of the auditory system (Mondor \& Zatorre, 1995), thereby allowing it to affect the processing of even somewhat distant visual targets. Alternatively, the effects of auditory cues on distant visual targets might arise from sensory or perceptual interactions because of the center-surround organization of the relatively large receptive fields of multisensory cells in the brain areas involved in the posterior attention network (see Stein \& Meredith, 1993).

\section{REFERENCES}

Bertelson, P., \& Aschersleben, G. (1998). Automatic visual bias of perceived auditory location. Psychonomic Bulletin \& Review, 5, 482 489.

Buchtel, H. A., \& Butter, C. M. (1988). Spatial attention shifts: Implications for the role of polysensory mechanisms using visual and tactile stimuli. Neuropsychologia, 26, 499-509.

Butter, C. M., Buchtel, H. A., \& SANTucci, R. (1989). Spatial attention shifts: Further evidence for the role of polysensory mechanisms using visual and tactile stimuli. Neuropsychologia, 27, 1231-1240.

Craft, J. L., \& Simon, J. R. (1970). Processing symbolic information from a visual display: Interference from an irrelevant directional cue. Journal of Experimental Psychology, 83, 415-420.

Driver, J., \& SPENCE, C. (1998). Attention and the crossmodal construction of space. Trends in Cognitive Sciences, 2, 254-262.

Farah, M. J., Wong, A. B., Monheit, M. A., \& Morrow, L. A. (1989). Parietal lobe mechanisms of spatial attention: Modality-specific or supramodal? Neuropsychologia, 27, 461-470.

Klein, R. [M.], Brennan, M., \& Gilani, A. (1987, November). Covert cross-modal orienting of attention in space. Paper presented at the annual meeting of the Psychonomic Society, Seattle.

Lu, C.-H., \& Proctor, R. W. (1995). The influence of irrelevant location information on performance: A review of the Simon and spatial Stroop effects. Psychonomic Bulletin \& Review, 2, 174-207.

MCDONALD, J. J., \& WARD, L. M. (1999). Spatial relevance determines facilitatory and inhibitory effects of auditory covert spatial orienting. Journal of Experimental Psychology: Human Perception \& Performance, 25, 1234-1252.
MCDonald, J. J., \& WARD, L. M. (2000). On the crossmodal orienting of attention. Manuscript submitted for publication.

MONDOR, T. A., \& Amirault, K. J. (1998). Effect of same- and different-modality spatial cues on auditory and visual target identification. Journal of Experimental Psychology: Human Perception \& Performance, 24, 745-755.

MONDOR, T. A., \& ZATORRE, R. J. (1995). Shifting and focusing auditory spatial attention. Journal of Experimental Psychology: Human Perception \& Performance, 21, 387-409.

PRoctor, R. W., \& Pick, D. F. (1998). Lateralized warning tones produce typical irrelevant-location effects on choice reactions. Psychonomic Bulletin \& Review, 5, 124-129.

SimoN, J. R. (1968). Effect of ear stimulated on reaction time and movement time. Journal of Experimental Psychology, 78, 344-346.

Simon, J. R., Acosta, E., JR., \& MeWaldt, S. P. (1975). Effect of locus of warning tone on auditory choice reaction time. Memory \& Cognition, 3, 167-170.

Simon, J. R., \& Craft, J. L. (1970). Effects of an irrelevant auditory stimulus on visual choice reaction time. Journal of Experimental Psychology, 86, 272-274.

SPENCE, C., \& DrIVER, J. (1996). Audiovisual links in endogenous covert spatial orienting. Journal of Experimental Psychology: Human Perception \& Performance, 22, $1005-1030$.

SPENCE, C., \& DrIVER, J. (1997). Audiovisual links in exogenous covert spatial orienting. Perception \& Psychophysics, 59, 1-22.

Spence, C., Nicholls, M. E. R., Gillespie, N., \& Driver, J. (1998). Cross-modal links in exogenous covert spatial orienting between touch, audition, and vision. Perception \& Psychophysics, 60, 544-557.

Stein, B. E., \& Meredith, M. A. (1993). The merging of the senses. Cambridge, MA: MIT Press.

Tassinari, G., Aglioti, S., Chelazzi, L., Peru, A., \& Berlucchi, G. (1994). Do peripheral non-informative cues induce early facilitation of target detection? Vision Research, 34, 179-189.

Tassinari, G., \& Berlucchi, G. (1995). Covert orienting to noninformative cues: Reaction time studies. Behavioural Brain Research, 71, 101-112.

WARD, L. M. (1994). Supramodal and modality-specific mechanisms for stimulus-driven shifts of auditory and visual attention. Canadian Journal of Experimental Psychology, 48, 242-259.

WARD, L. M., MCDonald, J. J., \& Golestani, N. (1998). Crossmodal control of attention shifts. In R. D. Wright (Ed.), Visual attention (pp. 232-268). New York: Oxford University Press.

WELCH, R. B., \& WARREN, D. H. (1986). Intersensory interactions. In K. R. Boff, L. Kaufman, \& J. P. Thomas (Eds.), Handbook of perception and human performance: Vol. I. Sensory processes and perception (pp. 1-36). New York: Wiley.

\section{NOTES}

1. For the sake of brevity, we use the term Simon effect to refer to an effect of irrelevant spatial information on any choice reaction time. However, the term usually has a more specific meaning, referring to an effect of irrelevant spatial information on nonspatial choice reaction times (Lu \& Proctor, 1995).

2. Spence and Driver (1997; Spence et al., 1998) have suggested that Ward's (1994) results might have reflected overt orienting because eye position was not strictly monitored. Unfortunately, Spence and Driver did not monitor eye position in their auditory cue-visual target experiment (Experiment 1), leading to the same alternative explanation for their results.

(Manuscript received October 27, 1998; revision accepted for publication September 29, 1999.) 